\title{
Exposure Therapy for Phobia Treatment
}

\author{
Ruiqi Guo
}

\author{
School of Psychology and Neuroscience, University of St.Andrews,Fife,KY16 9AJ,UK \\ 1368510583@qq.com
}

\begin{abstract}
Exposure therapy is used to curb various phobias and boost a healthy mental state of uoa person. The objective of this research study was to analyze how exposure therapy helps to overcome phobia. From the research study, it was found that exposure therapy involves any treatment that motivates the external systematic confrontation of the feared stimuli: like situations, objects or internal like situations and thoughts. From various studies it is evident that prolonged exposure is a very effective way of elimination of phobias and traumas. Exposure therapy aims at reducing a person's reaction of the fear to the stimulus. Four following prolonged exposures (PE) components were analyzed that is vivo exposure, applied muscle tension, virtual reality exposure and systematic desensitization. The study was deeply supported by through the study of the prolonged exposure. The study was anchored with theories such as emotional processing theory habituation theory and extension theory. Extinction theory involves gradual weakening of response conditioned which results to change in behavior or disappearing stops eventually. Emotional processing theory associative networks activates fear and information concerning the feared stimulus or escape. When presented in extreme, four becomes problem and gets in the way off functioning. Habituation involves two processes that are independent which interact to result to a final outcome of behavior. Various clinical and Veteran Health Administrations have come up with the training programs of prolonged exposure to professionals who help the patients with the phobia. The study adopted descriptive research design during the process analyzing data and data collection. Data was also cleaned and coded, finally the study would recommend that further studies be carried out to find out if the same results would be obtained.
\end{abstract}

Keywords: Exposure therapy, Phobias, Vivo exposure, Applied muscle tension, Virtual reality exposure, Systematic desensitization

\section{INTRODUCTION}

Exposure therapy involves any treatment that motivates the external systematic confrontation of the feared stimuli: like situations, objects or internal like situations and thoughts. Exposure therapy aims at reducing a person's reaction of the fear to the stimulus. Most therapists use a graded approach where the feared stimuli are targeted from the mildly feared to the strongly feared stimuli [1]. Therefore, an exposure hierarchy is ranked accordance to their anticipated reaction towards fear. Exposure therapy weakens the conditioned response through a repetitive exposure to the conditioned stimuli in absence of the unconditional stimuli. Design of exposure therapy is to encourage people to face the feared situations (imaginable or reality) and to remain in those situations [2]. The exposure applied greatly depends on the type of phobia. Phobia involves something a person fears the most. This type of disorder may impact a person's daily experiences and if not treated it may be severe. These phobias include: fears of snakes,
Acrophobia which is a fear of heights, Aerophobia flight fear and Gynophobia fear of dogs [3]. These phobias can be solved using exposure treatment. Exposure therapy has been proved to be a very effective of curbing phobias. This paper will analyze exposure treatment as a therapy to phobia. However, it is not very clear the factors that influence the exposure therapy but this paper discussed the current research in regard with the topic.

\section{RESEARCH QUESTIONS}

1. What is the effect of vivo exposure therapy for phobia?

2. What is the effect of applied muscle tension on phobia?

3. How does virtual reality exposure act on phobia?

4. How does systematic desensitization work on phobia treatment? 


\section{LITERATURE REVIEW}

Various research studies have been done by different authors to know the effectiveness of exposure therapy for phobia. Different theories analyzes have been studied to support the research. The literature review chapter is divided into theoretical framework which discusses the: habitation theory, extinction theory and emotional theory of state and empirical framework which discusses the four objectives of the study from different authors.

\subsection{Theoretical framework}

The following theories were studied to look deep into the concept of the exposure therapy to phobia treatment. The theories were habitation theory, extinction theory and emotional theory of state.

\subsection{Habitation theory}

The theory done by Maples-Keller (2020) states that after a repetition on presentation of stimuli, the response to the same stimuli will decrease [4]. For instance, when a person is initially exposed to cold water it may be cold, However as time passes and exposure continues, the water feels cold as the person adopts. The same way, where a person facing a certain fear-provoking stimuli is continuously exposed to exposure therapy, the patient experiences a natural reduction in the response to fear. Clinicians as for occurrence of habituation during the session, however research shows that there is effect of optimal treatment during learning sessions. According to the author habituation involves two processes that are independent which interact to result to a final outcome of behavior. He also stated that habituation of behavior is as a result of various mechanisms depending on stimulation time frame, the sensor pathway and processing level. According to the different mechanisms notions and in different pathways. More so the author stated that when a person learns that to present themselves or respond to a stimulus presented continuously without change, reward or punishment. Psychotherapy relays on habituation in phobia treatments. Examples include fear of being in a dark room. Once the person has habituated to this experience closer. At the end, the person will be habituated to the stimulus and they no longer experience fear.

\subsection{Extinction theory}

The theory of extinction carried out by Abramowitz (2013) discussed exposure therapy as a therapy treatment. From the classic model of conditioning where the unconditional stimulus is a person situation or place that caused fear like a dog bite. Through stimulus generation on process, reactions of fear become learned and elicited by other stimuli like non-dangerous dogs. Therefore, individuals who fear are motivated not to fear the conditional stimuli and gain avoidance behavior and believe that avoidance is relieve from the fear. The author explained that extinction theory involves gradual weakening of response conditioned which results to change in behavior or disappearing stops eventually [5]. For instance, if a person taught a dog to shake hands, the trick becomes less fascinating over time and when you stop rewarding that behavior and a person stops asking the dog forgets to shake hands. He also states that, coordinated stimuli ceases where presented done with no unconditioned stimulus. The same training a dog to feed after the bell is rung the dog salivates. But when the bell is rang and no food offered, the dog eventually stops associating bell with food and finally salivation stops. The author linked this training of dog with the same training to a person with phobia, when he is trained for $\mathrm{s}$ period of time he will eventually eliminate the phobia [5].

\subsection{Emotional theory of state}

Emotional processing theory as carried out by Baker (2010) states that fear is a network of stimuli stored in the memory like social gathering a person may get sweaty palms showing that the person is no good at socializing. [8] Therefore exposure to stimuli that provokes fear creates a new way of processing information. For instance, social interaction can be considered as a reward even when patients get sweaty palms.

According to the author emotional processing theory associative networks activates fear and information concerning the feared stimulus or escape [6]. When presented in extreme, four becomes problem and gets in the way off functioning. He also proposes that through emotional processing theory that is exposure may alter the relationship between the stimuli to networks. As a result, network that is not dangerous must be encoded.

\section{EMPIRICAL FRAMEWORK}

\subsection{Vivo exposure and phobia}

Study carried out by Soravia et al., (2014) shows that Vivo exposure involves confrontation of feared stimuli for instance; a person with spider phobia starts with phobia can start by reading plane crush stories and then later learn to talk about the actual flight. The authors argued that most phobias respond to Vivo exposure treatment as the study shows in vivo exposure, the exposure therapy is effective for phobia. The authors argued that through vivo exposure, the patient understands the consequences of their phobias are not reasonable. Thus patients become conditioned to the condition they have feared and the results of the same condition no longer provokes them to fear. They claimed that through vivo exposure, people effectively reduce anxiety and distress especially on people suffering from phobias. In this study the authors however argued that 
vivo exposure does not guarantee how a person will respond but the number of people helped by the program are more as compared to the others. The authors also states that it helps a person emotionally to process phobia events through helping a person to facing phobia or any other phobic behavior. After this therapy, a person can remember phobia images and memories safely without experiencing traumas [7].

\subsection{Applied Muscle Tension and Phobia}

Research carried out by Botella (2017) shows that applied muscle tension aids in treatment of a phobia 0 blood injection-injury. It uses a standard exposure as a technique and also uses muscle tension to respond to decline in blood pressure which may result to fainting. The author argued that exposure therapy is effective for phobia. Author further explained that muscle tension reduces vasovagal reactions through blood pressure maintenance and it is used to treat phobia. The author states that this method helps to prevent fainting or help the people who have fainted recover quickly. Applied Muscle tension involves testing a person's body muscles thus raising the blood pressure thus when a person's, blood pressure increases there is a reduced likelihood of fainting. A psychologist will focus on altering a person's psychology responses through a person practicing applied muscle releasing and tension when exposed to a certain phobia. In order to increase anxiety- evoking stimuli which is associated with the posed situation. The study is successful and has been used in patient treatment with specific phobias. It has helped many people deal with their anxiety therefore it is an effective exposure therapy for phobia. I conquer with the author in that exposure therapy can be a good remedy for phobia [8].

\subsection{Virtual reality exposure and phobia}

Study carried out by Garcia- Palacios (2011) shows that virtual reality exposure uses computer programs for phobia situation generation, like being on a plane taking off through association of computer graphics with body tracking devices for the individual to interact with that specific environment. Author also argued that various devices may be used to help in this form of therapy as following; body tracking devices, sensory input and other visual displays which guide a person to a computer generated environment. Research found that various phobias such as height, water, flying can be treated using the virtual reality exposure. All this has been evident showing that this form of therapy is effective. Through this study, a VR system that recognizes individual differences and also the system adjusts scenarios during simulation in accordance to the specified aim of treatment. The study also shows that in order to show that the study works, arachnophobia was used as a phobia. The system keeps an individual in a controlled need of therapist. Through all these processes used in therapy the phobia eventually ceases. In this study the author critically explained the effectiveness of exposure therapy as a treatment to phobia [9].

\subsection{Systematic desensitization and phobia}

Another study carried out by Lang and Lazovik (2013) shows how systematic desensitization entails exposing people with phobias to images ad pictures that evoke fear or actual phobia stimulus with relaxations to decrease the initial fear responses. The author also argued that this treatment is longer than vivo exposure. However, it helps in tackling phobia in a very effective way. The author stated that when fear or phobia starts becoming overwhelming and start interfering with a person's daily life, issues is more serious. Author explained that systematic desensitization exposes a person to the relaxation exercise where the person engages in certain activities to relax. The author stated that the combination of those two (exposure and relaxation) helps the person to face their fear. Through these process is extensive when a person's phobia is intense, it has been in use for a long period and has been effective. Thus as from the author's argument this supports my research in that exposure therapy is effective for phobia [10].

\section{METHODOLOGY}

\subsection{Research design}

The study adopted the use of description research design according to Creswell (2013).It involved information collection through interviews, observations, surveys, questionnaire to people [11]. The data collection methods helped study of exposure therapy as an effective treatment of therapy in that it provides data for analysis. The data was checked for completeness, mistakes and consistency. Description technique was used such as mean, frequency and percentages. Primary data was collected through the administration of questionnaire and interviews. The questionnaire were both open and closed ended.

\subsection{Reliability and validity tests for data collection}

Validity involves what an instrument measures and how well it measures. Reliability involves the faith a person has in the data collected and the degree in which a certain measuring tool controls the random of error.

\subsection{Results and findings}

Descriptive variables are vivo exposure, applied muscle tension, virtual reality exposure and systematic desensitization. From the response gathered $90 \%$ of the people stated that exposure therapy is effective for phobia. Therefore 
Table 1. The effectiveness of people who accepted the exposure therapy

\begin{tabular}{|l|l|l|}
\hline agreed & Disagreed & neutral \\
\hline $90 \%$ & $5 \%$ & $5 \%$ \\
\hline
\end{tabular}

From the people who had previously undergone the exposure therapy they agree $100 \%$ that the method is very effective for the treatment of phobia because their experience was $100 \%$ positive. The therapists from different areas confirmed that the best remedy for phobia treatment is exposure therapy since it helps the person to physically experience the situations I real life and after some time the results are recommendable. The people who Had taken their loved ones to undergo through the process of exposure therapy for treatment of phobia confirmed to us that the method was very effective since the phobia of the people had very effective in that it helped them to fully recover from phobia. $5 \%$ of the interviewed people who had not tried the method neither disagreed nor agreed but they said they could give it a try if the need arise in future. Remaining 5\% disagreed in tat the exposure therapy is not effective for phobia treatment although they never gave a valid reason. The findings above were guided by four objectives that is vivo exposure, applied muscle tension, virtual reality exposure and systematic desensitization. From majority of the findings it showed that these types of exposure helped curb certain phobias such as Arachnophobia, Ophidrophobia, Gynophobia and Acrophobia among many others. It also showed that results produced were effective therefore helping curb phobia. From the findings exposure therapy is effective for phobia. From the study I would recommend that clinics use exposure therapy to curb phobias.

\section{CONCLUSION}

This article serves as a literature review, summarizing more than ten articles on the effectiveness of exposure therapy for phobias. Introduced four different treatment methods in detail, include the treatment process they used and the final result, such as the impact on the patient.However, only four exposure therapy were analyzed namely vivo exposure, applied muscle tension, virtual reality, exposure and systematic desensitization. In order to ensure that the study of is exposure therapy effective for phobia is comprehensive, the records suggest further studies be done to investigate if exposure therapy is effective for phobia.

\section{REFERENCES}

[1] Neudeck, P., \& Wittchen, H. U. (Eds.). (2012). Exposure therapy: Rethinking the modelrefining the method. Springer Science \& Business Media.

[2] Klinger, E., Légeron, P., Roy, S., Chemin, I., Lauer,
F., \& Nugues, P. (2014). Virtual reality exposure in the treatment of social phobia. In Cybertherapy (pp. 91-119). IOS Press.

[3] Öst, L. G., Sterner, U., \& Fellenius, J. (2009). Applied tension, applied relaxation, and the combination in the treatment of blood phobia. Behavior Research and Therapy, 27(2), 109-121.

[4] Maples-Keller, J. L., S. A. (2020). Habituation.

[5] Abramowitz, J. S. (2013). The practice of exposure therapy: Relevance of cognitive-behavioral theory and extinction theory. Behavior therapy, 44(4), $548-558$

[6] Baker, A., (2010). Does habituation matter? Emotional processing theory and exposure therapy for acrophobia. Behaviour research and therapy, 48(11), 1139-1143.

[7] Soravia, L. M., Heinrichs, M., Winzeler, L., Fisler, M., Schmitt, W., Horn, H., \& de Quervain, D. J. F. (2014). Glucocorticoids enhance in vivo exposurebased therapy of spider phobia. Depression and anxiety, 31(5), 429-435.

[8] Botella, C. Recent progress in virtual reality exposure therapy for phobias: a systematic review. Current psychiatry reports, 19(7), 1-13.

[9] Garcia-Palacios, A., (2011). Redefining therapeutic success with virtual reality exposure therapy. CyberPsychology \& Behavior, 4(3), 341348.

[10] Lang, P. J., \& Lazovik, A. D. (2013). Experimental desensitization of phobia. The Journal of Abnormal and Social Psychology, 66(6), 519.

[11] Creswell, J. W., (2013). Research design (pp. 155179). Thousand Oaks, CA: Sage publications. 